\title{
Does chronic oral anticoagulation reduce in-hospital mortality among COVID-19 older patients?
}

\author{
Marcello Covino ${ }^{1,2}$ - Giuseppe De Matteis ${ }^{3}$ (D) Davide Della Polla ${ }^{1} \cdot$ Maria Livia Burzo ${ }^{4,5}$. Marco Maria Pascale ${ }^{1}$. \\ Michele Santoro $^{1}$. Raimondo De Cristofaro ${ }^{2,6}$. Antonio Gasbarrini ${ }^{2,3}$. Erica De Candia ${ }^{2,6}$. Francesco Franceschi ${ }^{1,2}$
}

Received: 18 April 2021 / Accepted: 22 June 2021 / Published online: 3 July 2021

(c) The Author(s), under exclusive licence to Springer Nature Switzerland AG 2021

\begin{abstract}
Background Patients hospitalized with COVID-19 experienced an increased risk of venous thromboembolism.

Aims To evaluate the effect of chronic oral anticoagulation (OAC) therapy, both with vitamin K antagonists (VKAs) or direct oral anticoagulants (DOACs), on prognosis of COVID-19 older patients.

Methods Single-center prospective study conducted in the Emergency Department (ED) of a teaching hospital, referral center for COVID-19 in central Italy. We evaluated all the patients $\geq 65$ years, consecutively admitted to our ED for confirmed COVID-19. We compared the clinical outcome of those who were on chronic OAC at ED admission with those who did not, using a propensity score matched paired cohort of controls. The primary study endpoint was all-cause in-hospital death. Patients were matched for age, sex, clinical comorbidities, and clinical severity at presentation (based on NEWS $\geq 6$ ). Study parameters were assessed for association to all-cause in-hospital death by a multivariate Cox regression analysis to identify independent risk factor for survival.

Results Although overall mortality was slightly higher for anticoagulated patients compared to controls (63.3\% vs $43.5 \%$, $p=0.012$ ), the multivariate adjusted hazard ratio (HR) for death was not significant (HR = $1.56[0.78-3.12] ; p=0.208)$. Both DOACs (HR 1.46 [0.73-2.92]; $p=0.283$ ) and VKAs (HR 1.14 [0.48-2.73]; $p=0.761$ ) alone did not affect overall survival in our cohort.

Conclusions Among older patients hospitalized for COVID-19, chronic OAC therapy was not associated with a reduced risk of in-hospital death. Moreover, our data suggest similar outcome both for patients on VKAs or in patients on DOACs.
\end{abstract}

Keywords COVID-19 . Older patients · Oral anticoagulation · Vitamin k antagonists · Direct oral anticoagulants

\section{Introduction}

Giuseppe De Matteis

giuseppe.dematteis@policlinicogemelli.it

1 Emergency Department, Fondazione Policlinico Universitario A. Gemelli IRCCS, Rome, Italy

2 Università Cattolica del Sacro Cuore, Roma, Italia

3 Department of Internal Medicine, Fondazione Policlinico Universitario A. Gemelli IRCCS, Rome, Italy

4 Emergency Department, Ospedale Generale M.G. Vannini, Istituto Figlie di San Camillo, Rome, Italy

5 Ospedale Pediatrico Bambin Gesù IRCCS, Rome, Italy

6 Department of Medicine and Translational Surgery, Fondazione Policlinico Universitario A. Gemelli IRCCS, Rome, Italy
The novel coronavirus designated SARS-CoV-2, that emerged in late 2019, has caused the current outbreak of respiratory illness named COVID-19 [1]. Although the vaccination campaigns have been started few months ago, the number of infected people and death toll is still on the rise worldwide [2].

Italy is facing one of the largest clusters of COVID-19, and mortality burden is extremely high [3], especially among older people and those with multiple comorbidities $[4,5]$. In particular, pre-existing cardiovascular diseases have proved to be one of the main predictors of poor prognosis in COVID-19 patients $[4,5]$.

Previous studies demonstrated that SARS-CoV-2 infection could lead to extensive vascular inflammation and endothelial dysfunction, with a consequent hypercoagulable 
state leading to the development of micro- and macrothrombosis, in vessels of both lungs and extrapulmonary organs [6]. Indeed, early reports had shown that hospitalized patients with COVID-19 experienced an increased risk of venous thromboembolism [7]. Similarly, recent evidences showed that the prevalence of macrovascular thrombotic complications are higher than expected in patients with COVID-19, mostly in the case of severe disease [8]. As a consequence, the role of a procoagulant state in the pathophysiology of severe COVID-19 has led physicians to use anticoagulation as a therapeutic option even if the possible beneficial role of the antithrombotic therapy in COVID-19 patients is a matter of an ongoing debate and definitive evidence is still lacking $[9,10]$.

However, since such hypercoagulable state could affect prognosis of patients hospitalized with COVID-19, the question has been raised whether chronic oral anticoagulation (OAC) therapy, in use for standard indications prior the SARS-CoV-2 infection, could have a protective role in reducing mortality or if these patients remain at high risk of death due to aging and multi-morbidity.

Thus, the aim of this study is to evaluate if chronic OAC therapy, either with vitamin K antagonists (VKAs) or direct oral anticoagulants (DOACs), could affect prognosis among patients aged 65 years or older hospitalized with COVID-19.

\section{Materials and methods}

\section{Study population and design}

This is a single-center, retrospective observational study, conducted in the emergency department (ED) of an urban teaching hospital, which is a referral center for COVID-19, in central Italy.

We evaluated all the patients $\geq 65$ years, consecutively admitted to our ED for confirmed COVID-19 from March 15th to December 31st, 2020. COVID-19 was diagnosed on the basis of the WHO interim guidance. We included in the analysis only patients with positive result on real-time reverse-transcriptase-polymerase-chain-reaction assay of nasal and pharyngeal swab specimens [11].

\section{Study variables}

The following information were evaluated:

- Demographic: age, sex.

- Clinical presentation at ED admission: fever, dyspnea, cough, diarrhea, confusion, ageusia/anosmia, asthenia/ myalgia.

- Clinical history and comorbidities. Hypertension, history of coronary artery disease (CAD), congestive heart fail- ure, cerebrovascular disease, dementia, diabetes, chronic obstructive pulmonary disease (COPD), chronic kidney disease, malignancy. Overall comorbidity presence was assessed by Charlson Comorbidity index (CCI).

- Radiological findings defined as: negative, interstitial involvement, and consolidative pneumonia.

- Physiological parameters at ED admission: body temperature, heart rate (HR), respiratory rate (RR), systolic blood pressure (SBP), level of consciousness assessed by the response on AVPU (Alert, Voice, Pain, Unresponsive) scale, peripheral oxygen saturation $\left(\mathrm{SpO}_{2}\right)$ in ambient air. Based on physiological parameters, the national early warning score (NEWS) was obtained for all patients [12].

- Laboratory values. For all patients, we evaluated total white cell blood (WBC) count, lymphocyte count, creatinine, blood urea nitrogen (BUN), lactate dehydrogenase $(\mathrm{LDH})$, serum fibrinogen, $\mathrm{C}$ reactive protein (CRP), and D-Dimer.

\section{Study design}

We selected all patients who have been continuously taking OAC therapy, either on VKAs or DOACs, for at least 1 month before ED admission. Consequently, patients noncompliant with OAC were excluded from the analysis.

Based on age, sex, comorbidity (categorized as $\mathrm{CCI}<3$ or $\mathrm{CCI} \geq 3$ ), and illness severity at admission (categorized as NEWS $<6$ or NEWS $\geq 6$ ), we obtained a matched cohort of controls by 1:1 propensity score matching (PSM). PSM was calculated by a logistic regression model using the nearest neighbor technique with no replacement, and with a caliper size of 0.2 to avoid poor matching. A detailed description of PSM analysis is described in supplementary materials (Appendix S1, Table S1, Figures S1, S2).

\section{Study endpoints}

The primary study endpoint was all-cause in-hospital death.

\section{Statistical analysis}

Continuous variables are reported as median [interquartile range], and are compared at univariate analysis by Mann-Whitney $U$ test. Categorical variables are reported as absolute number (percentage), and are compared by Chisquare test (with Fisher's test if appropriate).

Follow-up and length of hospital stay were calculated from the time of ED admission to 30 days or death. Evaluated parameters were assessed for association to all-cause in-hospital death by a univariate Cox regression analysis. Significant variables at univariate analysis were entered into a multivariate Cox regression model to identify 
independent risk factors for survival. For a better model fitting and hazard estimation, we categorized the continuous variables into dichotomous parameters (i.e. low/high). For each variable, we obtained the optimal dividing cut-off by Youden's index, performing a ROC curve analysis with respect to association with death.

To avoid model redundancy or overfitting, variables composed in CCI and NEWS were excluded from the analysis as separate items. Survival curves were estimated by the Kaplan-Meier methods. Association of factors to risk of intra hospital death was reported as hazard ratio (HR) [95\% confidence interval]. We considered significant a two-sided $p \leq 0.05$. Data were analyzed by SPSS v $25^{\circledR}$ (IBM, Armonk NY, USA).

\section{Results}

During the study period, 2666 confirmed COVID-19 patients were admitted to the ED. Among these 160 (6.0\%) were on anticoagulation therapy (42 [1.6\%] on VKA and 118 [4.4\%] on DOACs). Older adults $\geq 65$ years were 1407 (52.8\%), of whom 135 (9.6\%) were on anticoagulation (30 [2.1\%] on VKA and 105 [7.5\%] on DOACs). Among patients in the anticoagulated group $80(87 \%)$ were on oral anticoagulation due to atrial fibrillation.

After PSM based on age, sex, presence of $\geq 3$ comorbidities, and admission NEWS $\geq 6$, a total of 184 patients, 92 in anticoagulated group and 92 controls, were included in the study cohort.

\section{Comparison of the matched study groups}

Patients in the study cohort had a median age of 84 years [81-87], $66.8 \%$ of them had $\geq 3$ comorbidities, and $12.0 \%$ of them had a severe derangement of physiological parameters at admission (NEWS $\geq 6$ ) (Table 1). Anticoagulated group and controls were well balanced for age, sex, $\mathrm{CCI} \geq 3$, and NEWS $\geq 6$ at presentation (Table 1).

Patients in the anticoagulated group had a similar clinical presentation at admission, with the exception of a lower rate of fever $(67.4 \%$ vs $82.6 \%, p=0.017)$, and a higher rate of consolidation at chest X-ray $(45.6 \%$ vs $30.4 \%, p=0.09)$. Laboratory values were pretty similar in the two groups, with the exception of slightly higher LDH values found in the anticoagulated group (Table 1).

Although overall comorbidities were balanced in the two groups, anticoagulated patients had more cardiovascular comorbidities if compared to controls (Table 1).

\section{Survival analysis in anticoagulated patients vs. controls}

Overall, 60 patients (32.6\%) deceased, $38(41.3 \%)$ in the anticoagulated group and $22(23.9 \%)$ in controls $(p=0.012)$ (Table 1$)$.

Deceased patients were significantly older (85 [82-88] vs. 83 [80-87]; $p=0.012$ ), had less complaining of fever at presentation ( $60 \%$ vs. $82 \% ; p=0.001)$, and had a higher derangement of physiological parameters at ED admission, as expressed by a lower peripheral oxygen saturation, a higher respiratory rate and a lower systolic blood pressure (Table 2). As a result, deceased patients had a NEWS $\geq 6$ at admission in $23.3 \%$ of cases, compared to $6.5 \%$ in survived ones $(p=0.001$ ).

Consolidation at chest X-ray was present in $56.7 \%$ of deceased, compared to $29.0 \%$ of controls $(p=0.001)$. Most of laboratory values evaluated were significantly different in the deceased group. Deceased patients had higher WBC count, BUN, LDH, fibrinogen, CRP, D-Dimer, and lower lymphocyte count (Table 2).

A higher comorbidity rate was associated to increased mortality rate, as $81.7 \%$ of deceased patients had a $\mathrm{CCI} \geq 3$, compared to 59.7\% in survived ( $p=0.003$ ) (Table 2). Among the evaluated comorbidities mortality was significantly higher in patients with cerebrovascular disease and dementia (Table 2). No difference was found in terms of simultaneous aspirin/clopidogrel therapy.

\section{Multivariate analysis}

Prior to be entered into the multivariate models, continuous variables were dichotomized by ROC analysis Youden's index J. Cut off points were: age $\geq 85$ years; $\mathrm{WBC}>10,089$ / $\mathrm{mm}^{3}$; lymphocyte $\leq 1050 / \mathrm{mm}^{3} ;$ BUN $>32 \mathrm{mg} / \mathrm{dL}$; $\mathrm{LDH}>411 \mathrm{UI} / \mathrm{L} ; \mathrm{CRP}>50 \mathrm{mg} / \mathrm{dL}$; Fibrinogen $>549 \mathrm{mg} /$ $\mathrm{dL}$; D-Dimer $>818 \mathrm{ng} / \mathrm{mL}$.

When entered into a multivariate COX regression model, four of the evaluated factors emerged as independent risk factors for death, whereas anticoagulation status was not associated to a different outcome (Table 3; Fig. 1).

Although the median age of study cohort was high, age emerged as an independent risk factor for death, being death hazard ratio about doubled for age $\geq 85$ years (Table 3 ). Similarly, presence of consolidation at chest X-ray was associated to poor prognosis.

Since at univariate analysis, DOACs appeared to be associated to a worse prognosis compared to VKA, we ran a separate multivariate regression analysis including DOACs as factor. This latter analysis did not reveal an independent risk associated to DOACs assumption in our cohort (DOACs Wald $=1.153$, HR 1.46 [0.73-2.92]; $p=0.283$ ). 
Table 1 Demographic and clinical characteristics of enrolled patients at emergency department (ED) admission

\begin{tabular}{|c|c|c|c|c|}
\hline & $\begin{array}{l}\text { All cases } \\
n 184\end{array}$ & $\begin{array}{l}\text { Controls } \\
N 92\end{array}$ & $\begin{array}{l}\text { Anticoagulated } \\
N 92\end{array}$ & $p$ value \\
\hline Age & $84(81-87)$ & $84(79-88)$ & $84(81-86)$ & 0.724 \\
\hline Sex (male) & $92(50 \%)$ & $46(50 \%)$ & $46(50 \%)$ & 1.000 \\
\hline \multicolumn{5}{|l|}{ Clinical presentation } \\
\hline Fever & $138(75.0 \%)$ & $76(82.6 \%)$ & $62(67.4 \%)$ & 0.017 \\
\hline Dyspnea & $126(68.5 \%)$ & $58(63.0 \%)$ & $68(73.9 \%)$ & 0.112 \\
\hline Cough & $104(56.5 \%)$ & $53(57.6 \%)$ & $51(55.4 \%)$ & 0.766 \\
\hline Asthenia/myalgia & $15(8.2 \%)$ & $7(7.6 \%)$ & $8(8.7 \%)$ & 0.788 \\
\hline Confusion & $11(6.0 \%)$ & $4(4.3 \%)$ & $7(7.6 \%)$ & 0.536 \\
\hline Diarrhea & $7(3.8 \%)$ & $2(2.2 \%)$ & $5(5.4 \%)$ & 0.444 \\
\hline Ageusia/anosmia & $2(1.1 \%)$ & 0 & $2(2.2 \%)$ & 0.497 \\
\hline \multicolumn{5}{|l|}{ Physiological parameters } \\
\hline NEWS $\geq 6$ & $22(12.0 \%)$ & $11(12.0 \%)$ & $11(12.0 \%)$ & 1.000 \\
\hline Heart rate (beats/min) & $83(72-95)$ & $81(75-92)$ & $85(70-97)$ & 0.678 \\
\hline $\mathrm{SpO} 2(\%)$ in ambient air & $94(90-97)$ & $94(90-97)$ & $94(190-96)$ & 0.553 \\
\hline Respiratory rate (breaths/min) & $24(18-28)$ & $24(18-28)$ & $26(20-28)$ & 0.124 \\
\hline $\mathrm{SBP}(\mathrm{mmHg})$ & $128(112-140)$ & $129(120-140)$ & $128(106-140)$ & 0.172 \\
\hline \multicolumn{5}{|l|}{ Radiological findings } \\
\hline Negative & $44(23.9 \%)$ & $19(20.7 \%)$ & $25(27.2 \%)$ & \\
\hline Interstitial involvement & $70(38.0 \%)$ & $45(48.9 \%)$ & $25(27.2 \%)$ & 0.009 \\
\hline Consolidation & $70(38.0 \%)$ & $28(30.4 \%)$ & $42(45.6 \%)$ & \\
\hline \multicolumn{5}{|l|}{ Laboratory values } \\
\hline WBC count (cells/mm³) & $7680(5070-10,540)$ & 7400 (5025-9998) & $8040(5310-11,380)$ & 0.327 \\
\hline Lymphocyte (cells $/ \mathrm{mm}^{3}$ ) & $1050(740-1400)$ & $1000(692-1400)$ & $1050(770-1430)$ & 0.868 \\
\hline WBC/lymphocyte ratio & $7.34(4.56-11.31)$ & $6.47(3.94-10.13)$ & $7.91(4.92-11.41)$ & 0.175 \\
\hline Creatinine (mg/dL) & $1.01(0.78-1.47)$ & $1.05(0.70-1.70)$ & $1.01(0.80-1.39)$ & 0.976 \\
\hline BUN (mg/dL) & $25(19-37)$ & $24(18-38)$ & $25(19-36)$ & 0.868 \\
\hline LDH (UI/L) & $299(231-431)$ & $295(216-378)$ & $327(242-478)$ & 0.013 \\
\hline Fibrinogen (mg/dL) & $465(393-594)$ & $460(390-586)$ & 468 (399-597) & 0.717 \\
\hline CRP (mg/L) & $60(29-121)$ & $56(28-138)$ & $62(30-116)$ & 0.781 \\
\hline D-Dimer (ng/mL) & $1012(455-2481)$ & $986(433-2407)$ & $1012(444-2496)$ & 0.894 \\
\hline \multicolumn{5}{|l|}{ Clinical history-comorbidities } \\
\hline$\geq 3$ comorbidities & $123(66.8 \%)$ & $61(66.3 \%)$ & $62(67.4 \%)$ & 0.876 \\
\hline On aspirin or clopidogrel & $70(38.0 \%)$ & $41(44.6 \%)$ & $29(31.5 \%)$ & 0.068 \\
\hline Hypertension & $77(41.8 \%)$ & $34(37.0 \%)$ & $43(46.7 \%)$ & 0.179 \\
\hline History of CAD & $47(25.5 \%)$ & $9(9.8 \%)$ & $38(41.3 \%)$ & $<0.001$ \\
\hline Congestive heart failure & $43(23.4 \%)$ & $10(10.9 \%)$ & $33(35.0 \%)$ & $<0.001$ \\
\hline Cerebrovascular disease & $20(10.9 \%)$ & $3(3.3 \%)$ & $17(18.5 \%)$ & 0.001 \\
\hline Dementia & $27(14.7 \%)$ & $19(20.7 \%)$ & $8(8.7 \%)$ & 0.022 \\
\hline COPD & $32(17.4 \%)$ & $20(21.7 \%)$ & $12(13.0 \%)$ & 0.120 \\
\hline Diabetes & $34(18.5 \%)$ & $18(19.6 \%)$ & $16(17.4 \%)$ & 0.704 \\
\hline Chronic kidney disease & $28(15.2 \%)$ & $7(7.6 \%)$ & $21(22.8 \%)$ & 0.004 \\
\hline Malignancy & $4(2.2 \%)$ & 0 & $4(4.3 \%)$ & 0.121 \\
\hline \multicolumn{5}{|l|}{ Outcome } \\
\hline Deaths & $60(32.6 \%)$ & $22(23.9 \%)$ & $38(41.3 \%)$ & 0.012 \\
\hline
\end{tabular}

Controls were selected among all the consecutive patients evaluated in our ED after a 1:1 propensity score matching including age, sex, national early warning score (NEWS) $\geq 6$ at admission, and presence of $\geq 3$ comorbidities

Bold values denote statistical significance $(p \leq 0.05)$

$S p O 2$ peripheral oxygen saturation, $S B P$ systolic blood pressure, $C A D$ coronary artery disease, $C O P D$ chronic obstructive pulmonary disease, $W B C$ white blood cells, $L D H$ lactate dehydrogenase, $B U N$ blood urea nitrogen, $C R P C$ reactive protein, $N E W S$ National early warning score 
Table 2 Univariate analysis for the study endpoint

\begin{tabular}{|c|c|c|c|}
\hline & $\begin{array}{l}\text { Survived } \\
N 124\end{array}$ & $\begin{array}{l}\text { Deceased } \\
N 60\end{array}$ & $p$ value \\
\hline Age & $83(80-87)$ & $85(82-88)$ & 0.012 \\
\hline Sex (male) & $59(47.6 \%)$ & $33(55.0 \%)$ & 0.345 \\
\hline \multicolumn{4}{|l|}{ Anticoagulation therapy } \\
\hline Either on VKA or DOACs & $54(43.5 \%)$ & $38(63.3 \%)$ & 0.012 \\
\hline VKA & $11(8.9 \%)$ & $7(11.7 \%)$ & 0.550 \\
\hline DOACs & $43(34.7 \%)$ & $31(51.7 \%)$ & 0.028 \\
\hline \multicolumn{4}{|l|}{ ED presentation } \\
\hline Fever & $102(82.3 \%)$ & $36(60.0 \%)$ & 0.001 \\
\hline Dyspnea & $82(66.1 \%)$ & $44(73.3 \%)$ & 0.324 \\
\hline Cough & $104(62.1 \%)$ & $27(45.0 \%)$ & 0.028 \\
\hline Asthenia/myalgia & $13(10.5 \%)$ & $2(3.3 \%)$ & 0.097 \\
\hline Confusion & $6(4.8 \%)$ & $5(8.3 \%)$ & 0.349 \\
\hline Diarrhea & $7(5.6 \%)$ & 0 & 0.061 \\
\hline Ageusia/anosmia & 0 & $2(3.3 \%)$ & 0.497 \\
\hline \multicolumn{4}{|c|}{ Physiological parameters at admission } \\
\hline NEWS $\geq 6$ & $8(6.5 \%)$ & $14(23.3 \%)$ & 0.001 \\
\hline Heart rate (beats/min) & $82(73-96)$ & $84(70-94)$ & 0.993 \\
\hline $\mathrm{SpO} 2(\%)$ in ambient air & $94(91-97)$ & $93(88-97)$ & 0.034 \\
\hline Respiratory rate (breaths/min) & $22(18-25)$ & $27(20-29)$ & 0.021 \\
\hline $\mathrm{SBP}(\mathrm{mmHg})$ & $130(118-140)$ & $120(103-139)$ & 0.022 \\
\hline \multicolumn{4}{|l|}{ Radiological findings } \\
\hline Negative & $36(29.0 \%)$ & $8(13.3 \%)$ & \\
\hline Interstitial involvement & $52(41.9 \%)$ & $18(30.0 \%)$ & 0.001 \\
\hline Consolidation & $36(29.0 \%)$ & $34(56.7 \%)$ & \\
\hline \multicolumn{4}{|l|}{ Laboratory values } \\
\hline WBC (cells $/ \mathrm{mm}^{3}$ ) & 7220 (4970-9877) & $8365(5950-12,275)$ & 0.047 \\
\hline Lymphocyte (cells/mm³) & $1120(790-1460)$ & $905(682-1210)$ & 0.008 \\
\hline WBC/lymphocyte ratio & $6.12(4.13-9.71)$ & $9.64(5.66-14.68)$ & $<0.001$ \\
\hline Creatinine (mg/dL) & $1.03(0.78-1.41)$ & $0.99(0.76-1.82)$ & 0.740 \\
\hline BUN (mg/dL) & $23(17-31)$ & $30(22-47)$ & 0.004 \\
\hline LDH (UI/L) & $283(222-393)$ & $360(251-514)$ & 0.004 \\
\hline Fibrinogen (mg/dL) & $455(372-542)$ & $523(423-697)$ & 0.016 \\
\hline $\mathrm{CRP}(\mathrm{mg} / \mathrm{L})$ & $49(23-108)$ & $92(52-156)$ & $<0.001$ \\
\hline D-Dimer (ng/mL) & $803(370-1898)$ & $1817(905-4552)$ & $<0.001$ \\
\hline \multicolumn{4}{|l|}{ Clinical history-comorbidities } \\
\hline$\geq 3$ comorbidities & $74(59.7 \%)$ & $49(81.7 \%)$ & 0.003 \\
\hline On aspirin or clopidogrel & $48(38.7 \%)$ & $22(36.7 \%)$ & 0.789 \\
\hline Hypertension & $51(41.1 \%)$ & $26(43.3 \%)$ & 0.776 \\
\hline History of CAD & $29(23.4 \%)$ & $18(30.0 \%)$ & 0.335 \\
\hline Congestive heart failure & $24(19.4 \%)$ & $19(31.7 \%)$ & 0.064 \\
\hline Cerebrovascular disease & $5(4.0 \%)$ & $15(25.0 \%)$ & $<0.001$ \\
\hline Dementia & $12(9.7 \%)$ & $15(25.0 \%)$ & 0.006 \\
\hline COPD & $23(18.5 \%)$ & $9(15.0 \%)$ & 0.552 \\
\hline Diabetes & $23(18.5 \%)$ & $11(18.3 \%)$ & 0.972 \\
\hline Chronic kidney disease & $15(12.1 \%)$ & $13(21.7 \%)$ & 0.090 \\
\hline Malignancy & $1(0.8 \%)$ & $3(5.0 \%)$ & 0.067 \\
\hline
\end{tabular}

Bold values denote statistical significance $(p \leq 0.05)$

$S p O 2$ peripheral oxygen saturation, $S B P$ systolic blood pressure, $C A D$ coronary artery disease, $C O P D$ chronic obstructive pulmonary disease, $W B C$ white blood cells, $L D H$ lactate dehydrogenase, $B U N$ blood urea nitrogen, $C R P \mathrm{C}$ reactive protein, $V K A$ vitamin $\mathrm{K}$ antagonists, $D O A C$ direct-acting oral anticoagulant 
Table 3 Multivariate analysis (Cox regression model) of significant factor associate to survival at univariate analysis
Fig. 1 Cumulative survival rate in anticoagulated patients vs controls. Once corrected for clinical and demographic characteristics at admission death risk was similar for anticoagulated and controls (anticoagulation $\mathrm{HR}=1.56$ [0.78-3.12]; $p=0.208)$

\begin{tabular}{llll}
\hline Factor & Wald & $\begin{array}{l}\text { Hazard ratio [95\% confi- } \\
\text { dence interval] }\end{array}$ & $\begin{array}{l}\text { Multivariate } \\
p \text { value }\end{array}$ \\
\hline Either on VKA or DOACs & 0.531 & $1.27[0.67-2.40]$ & 0.466 \\
Age $\geq 85$ years & 3.940 & $1.78[1.01-3.15]$ & $\mathbf{0 . 0 4 7}$ \\
NEWS $\geq 6$ at admission & 0.700 & $1.34[0.67-2.66]$ & 0.576 \\
CCI $\geq 3$ & 2.358 & $1.70[0.86-3.35]$ & 0.125 \\
Consolidation at chest X-ray & 4.546 & $1.87[1.05-3.33]$ & $\mathbf{0 . 0 3 3}$ \\
WBC/lymphocyte $>4.94$ & 0.925 & $1.42[0.69-2.91]$ & 0.336 \\
Blood urea nitrogen $>32 \mathrm{mg} / \mathrm{dL}$ & 0.930 & $1.33[0.74-2.40]$ & 0.335 \\
Lactate dehydrogenase $>411 \mathrm{UI} / \mathrm{L}$ & 0.008 & $0.97[0.52-1.83]$ & 0.931 \\
C reactive protein $>50 \mathrm{mg} / \mathrm{dL}$ & 2.166 & $1.69[0.84-3.42]$ & 0.141 \\
Fibrinogen $>549 \mathrm{mg} / \mathrm{dL}$ & 0.013 & $1.03[0.58-1.82]$ & 0.909 \\
D-Dimer $>818 \mathrm{ng} / \mathrm{mL}$ & 5.170 & $2.70[1.15-6.36]$ & $\mathbf{0 . 0 2 3}$ \\
\hline
\end{tabular}

Cut off values for continuous variables were chosen according to receiver operating characteristic (ROC) analysis Youden index $\mathrm{J}$

Bold values denote statistical significance $(p \leq 0.05)$

NEWS National Early Warning Score, VKA vitamin K antagonists, DOAC direct-acting oral anticoagulant, CCI Charlson comorbidity index

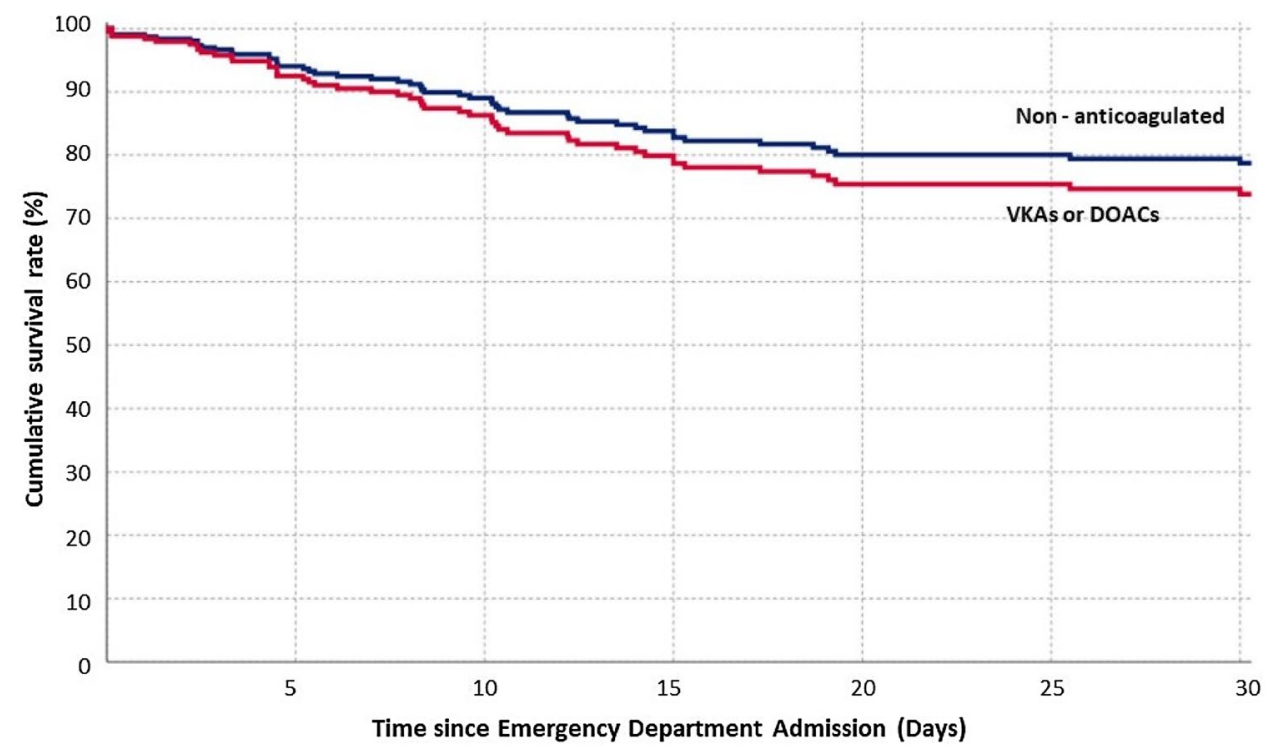

When the same analysis was made for patients on VKA, the results were similar (VKAs Wald $=0.092$, HR 1.14 [0.48-2.73]; $p=0.761)$.

\section{Discussion}

The main finding of present study is that among older patients $\geq 65$ years hospitalized for COVID-19, chronic OAC therapy was not associated with a reduced risk of inhospital death. Indeed, correcting the analysis for baseline clinical covariates and disease severity at ED admission, these patients have a similar in-hospital all-cause mortality compared to non-anticoagulated controls. Moreover, noticeably, our data suggest similar outcome both for patients on VKAs or in patients on DOACs.

Patients hospitalized with COVID-19 appear to be at higher risk of venous thrombosis, due to the interaction between coagulation and virus-related inflammation [13]. The resulting procoagulant state is mediated by the release inflammatory cytokines [Interleukin 6 (IL-6), Interleukin-8 (IL-8)], tumor necrosis factor $\alpha$ (TNF- $\alpha$ ), and chemokines that causes the activation of epithelial cells, monocytes, and neutrophils [14]. Alternatively, it has been postulated that endothelial cells can be directly infected through the ACE-2 
receptor, leading to endothelial activation which triggers the coagulation cascade. The following activation of platelets and protease-activated receptors elicit a highly proinflammatory state, starting up the local coagulation [15].

Thus, due to the close relationship between SARSCoV-2 infection and hypercoagulability, and the known increased thrombotic risk in hypoxic COVID-19 patients [16] antithrombotic therapy is often prescribed as a therapeutic option both for severe and mild cases. In the meanwhile, several ongoing randomized controlled trials are trying to evaluate doses, treatment duration, and potential effectiveness of anticoagulant therapy on the full spectrum of COVID-19 patients [17].

However, to date is still debated if chronic anticoagulation may act as a protective factor in patients already on treatment affected by COVID-19 or, conversely, the pro-thrombotic conditions which had led to chronic anticoagulation are per se associated to a poor prognosis $[9,10]$. Therefore, since anticoagulated patients are often older and have multiple comorbidities, this retrospective study was designed to correct for both demographic and clinical covariates, focusing on patients $\geq 65$ years who are at increased risk of poor prognosis if hospitalized with COVID-19. Moreover, our analysis was corrected for disease severity at presentation using the NEWS, that showed a good performance in risk stratification of COVID-19 older patients [18, 19].

The analysis of our cohort, corrected for comorbidities, age, sex, and clinical severity at hospital admission revealed a similar outcome for patients on chronic OAC compared to matched controls. Our findings are similar to those of another cohort study enrolling COVID-19 patients admitted to four different Italian institutions, investigating whether pre-hospitalization OAC or in-hospital heparin treatment could have a protective role among COVID-19 patients. This latter study showed that pre-hospitalization OAC treatment was ineffective in reducing mortality rate [20]. Similarly, a further and more recent report on a large cohort of 28,000 Sars-CoV-2 infected patients, found no association with decreased mortality, hospitalization or mechanical ventilation in patients on $\mathrm{OAC}$ or antiplatelets preceding SARS-CoV-2 infection diagnosis [21]. However, it should be pointed out that this study comprised only 304 anticoagulated patients, 215 of which $\geq 60$ years old. Likewise, DOACs use was not found to be associated with a different risk of severe disease or mortality in a population-based Swedish study on 103,703 patients on DOAC compared to 355,699 non-use controls [22].

However, several other reports investigating the effect of chronic OAC therapy on outcomes in patients hospitalized with COVID-19 showed different and contrasting results.

On one hand, a more recent study suggested that prior OAC treatment was associated to worse prognosis in COVID-19 [23]. This latter report, similar to the present study, made a full correction for patients' comorbidities using CCI in a PSM paired analysis. However, while the PSM was corrected for age, sex, and comorbidities, the study cohort was not adjusted for clinical severity at presentation, thus leaving the possibility that a worse outcome is just due to a more severe disease status in the OAC group.

On the other hand, another Italian retrospective population-based study, suggested that among patients $\geq 65$ years with COVID-19, those on OAC for atrial fibrillation could be at lower risk of all-cause mortality compared to their propensity score matched non-anticoagulated counterpart. Howbeit, this conclusion is questionable because among OAC patients the adjusted HR for all-cause mortality missed statistical significance [24]. Analogously, a German retrospective cohort study investigating the impact of OAC on clinical outcomes in patients hospitalized with coronavirus disease 2019, showed that a therapy with both DOACs or VKAs, but not with antiplatelet therapy, was associated with improved clinical outcomes [25]. Likewise, a recent secondary analysis on the database of a multicenter French cohort, suggested that prior OAC was associated to better prognosis in COVID-19 patients, in terms of both reduced intensive care unit admission and overall mortality [26]. However, this study yield contrasting results, since while prior OAC was associated to better prognosis, therapeutic or prophylactic low- or high-dose anticoagulation started during hospitalization were not associated with any of the outcomes [26]. Moreover, although authors corrected their analysis for clinical comorbidities, only cardiovascular factors associated to anticoagulation were available, and therefore, most of comorbidities frequent in older adults were not available for analysis adjustment. In addition, the analysis was not fully corrected for clinical severity at admission.

In addition, we should consider that the majority of patients in our cohort were on OAC due to atrial fibrillation. A recent paper by Mountantonakis et al. demonstrated that atrial fibrillation was an independent predictor of in-hospital mortality in patients admitted with SARS-CoV-2 infection [27]. Therefore, we cannot exclude that this clinical condition was by itself responsible for an increased risk of death, which could have masked a possible protective effect of oral anticoagulation, influencing our results.

The present study also confirm that age is a prominent risk factor for death in patients hospitalized with COVID19 [1], as previously reported for patients $\geq 80$ years [28], representing the majority of patients evaluated in our cohort. Indeed, increasing age is by itself a risk factor for overall COVID-19 in-hospital death, independent from clinical covariates, comorbidities and laboratory findings [29]. Furthermore, deceased patients in our study group demonstrated several laboratory findings commonly highlighted in severe COVID-19 patients (Table 2) [1]. Interestingly, only an elevated D-dimer emerged as independent risk factor for death. 
Although an elevated D-dimer is a common feature of severe COVID-19 [30], our finding suggests that also in anticoagulated patients the excessive activation of fibrin degradation is a marker of severe disease.

\section{Study limitations}

Our study presents several limitations. First, our limited sample may have reduced the power of the analysis. Second, the study was conducted only in a single institution and, even if it is a large COVID-19 referral center, our result could not be generalizable. Third, we did not correct our analysis for anticoagulation regimen during hospitalization. However, this latter finding is mitigated by the singlecenter nature of present study, which limit the therapeutic variability in our cohort. Finally, since our study design was focused on all-cause mortality, we did not evaluate the occurrence of thrombotic complications in our cohort during hospitalization.

\section{Conclusions}

Among older patients hospitalized for COVID-19, chronic treatment with OAC was not associated with a reduced risk of in-hospital death. Moreover, our data suggest similar outcome both for patients on VKAs or in patients on DOACs. However, further and larger studies are needed to validate these finding and to shed a definitive light on the possible benefit of therapeutic anticoagulation in older patients hospitalized with COVID-19.

Supplementary Information The online version contains supplementary material available at https://doi.org/10.1007/s40520-021-01924-w.

Author contributions MC: conceptualization, methodology, formal analysis, writing — review and editing. GDM: conceptualization, validation, visualization, writing — original draft, writing - review and editing. DAD and MLB: validation, visualization, writing — original draft, writing - review and editing. MMP AND MS: software, data curation RDC, EDC, AG: writing — review and editing. FF: supervision.

Funding No funding was received to assist with the preparation of this manuscript.

Availability of data and materials The datasets generated during and/or analyzed during the current study are available from the corresponding author on reasonable request.

\section{Declarations}

Conflict of interests The authors have no financial or proprietary interests in any material discussed in this article.
Statement of human and animal rights The study was conducted in accordance with the principles expressed in the Declaration of Helsinki and was approved by the local ethical committee (\#001705520).

Informed consent Informed consent was obtained from all individual participants included in the study.

Consent to participate Informed consent was obtained from all individual participants included in the study.

Consent to publish Patients signed informed consent regarding publishing their data.

\section{References}

1. Guan WJ, Ni ZY, Hu Y et al (2020) Clinical characteristics of coronavirus disease 2019 in China. N Engl J Med 382:1708-1720. https://doi.org/10.1056/NEJMoa2002032

2. World Health Organization. WHO Coronavirus Disease (COVID19) Dashboard. Available from: https://covid19.who.int/. Accessed on 12 Mar 2021

3. Ministero della Salute. COVID-19-situazione in Italia. http:// www.salute.gov.it/portale/nuovocoronavirus/. Accessed 12 Mar 2021

4. Clerkin KJ, Fried JA, Raikhelkar J et al (2020) Coronavirus disease 2019 (COVID-19) and cardiovascular disease. Circulation 141:1648-1655. https://doi.org/10.1161/CIRCULATIONAHA. 120.046941

5. European Society of Cardiology. ESC Guidance for the Diagnosis and Management of CV Disease during the COVID-19 Pandemic [last updated on 10 June 2020]. Available from: https://www.escar dio.org/Education/COVID-19-and-Cardiology/ESC-COVID-19Guidance

6. Zhou B, Zhao W, Feng R et al (2020) The pathological autopsy of coronavirus disease 2019 (COVID-2019) in China: a review. Pathog Dis 78:ftaa026. https://doi.org/10.1093/femspd/ftaa026

7. Cui S, Chen S, Li X et al (2020) Prevalence of venous thromboembolism in patients with severe novel coronavirus pneumonia. J Thromb Haemost 18:1421-1424. https://doi.org/10.1111/jth. 14830

8. Klok FA, Kruip MJHA, van der Meer NJM et al (2020) Confirmation of the high cumulative incidence of thrombotic complications in critically ill ICU patients with COVID-19: an updated analysis. Thromb Res 191:148-150. https://doi.org/10.1016/j.thromres. 2020.04.041

9. Connors JM, Levy JH (2020) COVID-19 and its implications for thrombosis and anticoagulation. Blood 135:2033-2040. https:// doi.org/10.1182/blood.2020006000

10. Maldonado E, Tao D, Mackey K (2020) Antithrombotic therapies in COVID-19 disease: a systematic review. J Gen Intern Med 35:2698-2706. https://doi.org/10.1007/s11606-020-05906-y

11. World Health Organization. Clinical management of severe acute respiratory infection when novel coronavirus (2019-nCoV) infection is suspect: interim guidance. Available from: https://www. who.int/emergencies/diseases/novel-coronavirus-2019/technicalguidance. Accessed 20 Mar 2020

12. McGinley A, Pearse R (2012) A national early warning score for acutely ill patients. BMJ 345:e5310. https://doi.org/10.1136/bmj. e5310 
13. Schulman S, Hu Y, Konstantinides S (2020) Venous thromboembolism in COVID-19. Thromb Haemost 120:1642-1653. https:// doi.org/10.1055/s-0040-1718532

14. Mehta P, McAuley DF, Brown M, HLH Across Speciality Collaboration, UK et al (2020) COVID-19: consider cytokine storm syndromes and immunosuppression. Lancet 395:1033-1034. https://doi.org/10.1016/S0140-6736(20)30628-0

15. Abou-Ismail MY, Diamond A, Kapoor S et al (2020) The hypercoagulable state in COVID-19: Incidence, pathophysiology, and management. Thromb Res 194:101-115. https://doi.org/10.1016/j. thromres.2020.06.029

16. Waite AAC, Hamilton DO, Pizzi R et al (2020) Hypercoagulopathy in severe COVID-19: implications for acute care. Thromb Haemost 120:1654-1667. https://doi.org/10.1055/s-0040-17214 87

17 Talasaz AH, Sadeghipour P, Kakavand H et al (2021) Recent randomized trials of antithrombotic therapy for patients with COVID-19: JACC state-of-the-art review. J Am Coll Cardiol 77:1903-1921. https://doi.org/10.1016/j.jacc.2021.02.035

18. Covino M, Sandroni C, Santoro M et al (2020) Predicting intensive care unit admission and death for COVID-19 patients in the emergency department using early warning scores. Resuscitation 156:84-91. https://doi.org/10.1016/j.resuscitation.2020.08.124

19. Covino M, De Matteis G, Burzo ML et al (2021) Predicting inhospital mortality in COVID-19 older patients with specifically developed scores. J Am Geriatr Soc 69:37-43. https://doi.org/10. 1111/jgs. 16956

20. Schiavone M, Gasperetti A, Mancone M et al (2021) Oral anticoagulation and clinical outcomes in COVID-19: an Italian multicenter experience. Int J Cardiol 323:276-280. https://doi.org/10. 1016/j.ijcard.2020.09.001

21. Ho G, Dusendang JR, Schmittdiel J et al (2021) Association of chronic anticoagulant and antiplatelet use on disease severity in SARS-COV-2 infected patients. J Thromb Thrombolysis 1:1-6. https://doi.org/10.1007/s11239-021-02383-w

22. Flam B, Wintzell V, Ludvigsson JF et al (2021) Direct oral anticoagulant use and risk of severe COVID-19. J Intern Med 289:411419. https://doi.org/10.1111/joim.13205
23. Rivera-Caravaca JM, Núñez-Gil IJ, Vivas D et al (2021) Clinical profile and prognosis in patients on oral anticoagulation before admission for COVID-19. Eur J Clin Invest 51:e13436. https:// doi.org/10.1111/eci.13436

24. Denas G, Gennaro N, Ferroni E et al (2021) Reduction in all-cause mortality in COVID-19 patients on chronic oral anticoagulation: a population-based propensity score matched study. Int J Cardiol 329:266-269. https://doi.org/10.1016/j.ijcard.2020.12.024

25. Fröhlich GM, Jeschke E, Eichler U et al (2021) Impact of oral anticoagulation on clinical outcomes of COVID-19: a nationwide cohort study of hospitalized patients in Germany. Clin Res Cardiol 8:1-10. https://doi.org/10.1007/s00392-020-01783-x

26. Chocron R, Galand V, Cellier J et al (2021) Anticoagulation prior to hospitalization is a potential protective factor for COVID-19: insight from a French multicenter cohort study. J Am Heart Assoc 8:e018288. https://doi.org/10.1161/JAHA.120.018624

27. Mountantonakis SE, Saleh M, Fishbein J, Northwell COVID-19 Research Consortium et al (2021) Atrial fibrillation is an independent predictor for in-hospital mortality in patients admitted with SARS-CoV-2 infection. Heart Rhythm 18:501-507. https:// doi.org/10.1016/j.hrthm.2021.01.018

28. Covino M, De Matteis G, Polla DAD et al (2021) Predictors of inhospital mortality AND death risk stratification among COVID-19 patients aged $\geq 80$ years old. Arch Gerontol Geriatr 95:104383. https://doi.org/10.1016/j.archger.2021.104383

29. Zhang XM, Jiao J, Cao J et al (2021) Frailty as a predictor of mortality among patients with COVID-19: a systematic review and meta-analysis. BMC Geriatr 21:186. https://doi.org/10.1186/ s12877-021-02138-5

30. Tang N, Bai H, Chen X et al (2020) Anticoagulant treatment is associated with decreased mortality in severe coronavirus disease 2019 patients with coagulopathy. J Thromb Haemost 18:10941099. https://doi.org/10.1111/jth.14817

Publisher's Note Springer Nature remains neutral with regard to jurisdictional claims in published maps and institutional affiliations. 